\title{
Alternatively Polarized Macrophages Regulate the Growth and Differentiation of Ependymal Stem Cells through the SIRT2 Pathway
}

\author{
Yonggang $\mathrm{Ma}^{1}$, Ming Deng ${ }^{1}$, Xiao-qi Zhao ${ }^{2}$ and Min Liu ${ }^{3 *}$ \\ ${ }^{1}$ Department of Orthopaedics, Renmin Hospital, Wuhan University, Wuhan 430060, ${ }^{2}$ Department of Pharmacology, School \\ of Basic Medical Sciences, Wuhan University, Wuhan 430071, ${ }^{3}$ Department of Immunology, School of Basic Medical Sciences,
} Wuhan University, Wuhan 430071, China

\begin{abstract}
Ependymal stem cells (EpSCs) are dormant stem cells in the adult spinal cord that proliferate rapidly and migrate to the site of injury after spinal cord injury (SCI). Although they can differentiate into neurons under appropriate conditions in vitro, EpSCs mainly differentiate into astrocytes in vivo. Our previous study confirmed that alternatively polarized macrophages (M2) facilitate the differentiation of EpSCs towards neurons, but the detailed mechanism remains elusive. In the present study, we found that M2 conditioned medium could upregulate the expression of Sirtuin 2 (SIRT2) in EpSCs in vitro through the BDNF/TrkB-MEK/ERK signaling pathway. As an important deacetylase, SIRT2 deacetylated stable Ac- $\alpha-$ tubulin (Acetyl alpha Tubulin) in microtubules and thus promoted EpSC differentiation into neurons. The present study provides a theoretical basis and a new way to improve neural recovery, such as regulating the growth and differentiation of EpSCs by increasing the proportion of M2 cells in the local microenvironment or upregulating the expression of SIRT2 in EpSCs.
\end{abstract}

Key words: Alternatively polarized macrophages, Ependymal stem cells, SIRT2

\section{INTRODUCTION}

Disruption of neural conduction pathways due to necrosis of neural tissues accounts for the paraplegia caused by spinal cord injury (SCI). Replacement of necrotic tissue with newborn nerve cells in an attempt to reconstruct conduction pathways is a promising strategy for neural rehabilitation following SCI. Various stem cells have been used as seed cells for transplantation and have shown some advantageous effects in vivo [1-3]. As one of representative cell type, ependymal stem cells (EpSCs) have received increasing attention in recent years.

Submitted November 27, 2019, Revised April 15, 2020,

Accepted April 16, 2020

${ }^{*}$ To whom correspondence should be addressed.

TEL: 86-18271910659, FAX: 86-2768759986

e-mail:1liumin2002@163.com
EpSCs are dormant stem cells in the adult spinal cord that proliferate rapidly and migrate to the site of injury after SCI $[4,5]$. Although they can differentiate into neurons in vitro, they mainly differentiate into astrocytes in vivo [6], which are unfavorable for neural regeneration. Some reports have demonstrated that neuroinflammation in the microenvironment plays a key role in regulating EpSCs growth and differentiation $[7,8]$. Our previous studies [9] confirmed that macrophages with different activation states (including M1 and M2) at sites of local injury affected the growth and differentiation of EpSCs. Specifically, M2 conditioned medium facilitated EpSCs differentiation towards neurons. The mechanism by which M2 acts on EpSCs in vivo, however, remains elusive.

In recent years, sirtuin deacetylases have emerged as candidate therapeutic targets for many human diseases, including metabolic and age-dependent neurological disorders [10]. In non-neuronal cells, Sirtuin 2 (SIRT2) has been shown to function as a tubulin 
deacetylase and a key regulator of cell division and differentiation [11]. Maxwell et al. [12] reported abundant and preferential expression of SIRT2 in the mammalian central nervous system, which correlated with reduced $\alpha$-tubulin acetylation in primary mouse cortical neurons and suggested that the brain-enriched species of SIRT2 may function as the predominant microtubule deacetylases in mature neurons. In another study, neural-specific protein expression (NF-M and MAP-2) was reduced following SIRT2 blockade in human bone marrow-mesenchymal stem cells (hBM-MSCs) [13]. In the present study, we aimed to explore whether M2 participates in regulating the growth and differentiation of EpSCs through the SIRT2 signaling pathway.

\section{MATERIALS AND METHODS}

\section{EpSC isolation and sorting}

Seven-to-nine-week-old male C57BL/6 (B6) mice were purchased from Hubei Provincial Center for Disease Control and Prevention (Wuhan, China). B6/SIRT2 ${ }^{-/}$mice were purchased from Shanghai Laboratory Animal Center (Shanghai, China). EpSCs were isolated and identified as described previously [9]. In brief, after a laminectomy, spinal cord tissue was harvested and cut into $1 \mathrm{~mm}^{3}$ pieces, enzymatically dissociated in a solution containing $0.01 \%$ papain and $0.01 \%$ DNase I (Worthington, Biochemicals, $\mathrm{NJ}$, USA) for $1 \sim 2 \mathrm{~h}$ at $37^{\circ} \mathrm{C}$, and mechanically dissociated into a cell suspension. Then, the suspensions were incubated with an antibody mix containing CD133-PE (1:100, clone 13A4, eBioscience, CA, USA) and CD24-fluorescein isothiocyanate (FITC; 1:500, clone M1/69, BD Biosciences, CA, USA) for $30 \mathrm{~min}$ on ice. Cells were sorted with a FACSDiva flow cytometer (BD Biosciences).

\section{Macrophage isolation, culture, and polarization}

M1 and M2 were generated as described previously [9]. In brief, bone marrow cells were isolated from the femurs of B6 mice or B6/ $\mathrm{BDNF}^{-/}$mice. The cells $\left(5 \times 10^{6}\right)$ were seeded onto $100-\mathrm{mm}$ sterile nontissue culture-treated polystyrene petri dishes (BD Biosciences with $12 \mathrm{ml}$ of R10 cell culture medium (RPMI 1640 [Corning, NY, USA] with 10\% fetal bovine serum [FBS; Thermo Fisher, MA, USA], 1\% penicillin/streptomycin [Thermo Fisher], and 1\% 4-[2-hydroxyethyl]-1-piperazineethanesulfonic acid HEPES [Sigma Aldrich, MO, USA]). The cells were stimulated to differentiate into M0 with $20 \mathrm{ng} / \mathrm{ml}$ macrophage colony-stimulating factor (M-CSF; eBioscience). Then, the cells were cultured at $37^{\circ} \mathrm{C}$ and $5 \% \mathrm{CO}_{2}$ for 6 days and were fed every third day. To obtain M1 cells, on day 7 of culture, we stimulated the M0 cells with $50 \mathrm{ng} / \mathrm{ml}$ interferon- $\gamma$ (IFN- $\gamma$; R\&D Systems, MN, USA) +20 $\mathrm{ng} / \mathrm{ml}$ lipopolysaccharide (LPS; Sigma-Aldrich). To obtain M2 cells, we stimulated the M0 cells with $20 \mathrm{ng} / \mathrm{ml}$ interleukin-4 (IL4; BD Biosciences). The cells were harvested on day 8. M1 and M2 were identified by western blot and RT-PCR analyses as described previously [9].

\section{Transwell coculture of EpSCs and macrophages}

A transwell system was used to evaluate the influence of polarized macrophages on EpSCs according to our previous method [9]. Macrophages were cultured on 24-well plate inserts (1-mm pore size, Corning, NY, USA) with EpSCs preplated on the bottom wells. In brief, the polarized macrophages (M0, M1 or M2) were washed with PBS, resuspended in B27 medium, and subsequently added to the inserts at an initial seeding density of $5 \times 10^{5}$ cells per insert. The inserts were placed on a 24 -well plate that contained EpSCs at $5 \times 10^{5}$ cells per well (bottom well). The bottom wells were precoated with poly-L-lysine (PLL; Sigma-Aldrich,). The cells were cultured in medium (B27 [w/o vitamin A, 1×], L-glutamine [2 mM], penicillin [100 U/ml], streptomycin [100 $\mu \mathrm{g} / \mathrm{ml}]$ [all from Invitrogen, CA, USA $]$, and partricin [0.5 $\mu \mathrm{g} / \mathrm{ml}$; Sigma-Aldrich]) for $3 \sim 7$ days. The macrophages in the inserts were changed every 3 days. The EpSCs were collected and used for immunostaining and western blotting assays according to the experimental scheme.

\section{Surgery and postoperative care}

Male B6 mice ( $\mathrm{n}=12$, weight 18 20 g) were allocated into the SCI group and the sham operation group. WT mice or $\mathrm{BDNF}^{-/}$mice ( $\mathrm{n}=6$, weight $18 \sim 20 \mathrm{~g}$ ) were deeply anaesthetized using $1.5 \%$ isoflurane with oxygen, and the surgical area was then shaved and sterilized with isopropanol and betadine. Buprenorphine $(0.05 \mathrm{mg} /$ $\mathrm{kg}$ ) and Baytril (5 mg/kg) were given before the surgery. A midline incision was made to expose the vertebral column, and a laminectomy was then performed at the T8-T9 level. The mice received a severe mid-thoracic (T8-T9) crush injury using Dumont-type forceps with a spacer of $0.2 \mathrm{~mm}$. Animals in the sham operation group underwent laminectomy only. Postoperative care included buprenorphine and Baytril administration for 2 days after surgery. Manual voiding of the bladders was performed twice daily until the bladder function returned.

The care, use and treatment of mice in this study were in strict agreement with international guidelines for the care and use of laboratory animals. This study was approved by the Animal Ethics Committee of the Medical School of Wuhan University (2018M53RMYD).

\section{Immunohistochemistry}

On days 1, 3, and 7 postinjury, 3 mice from each group were sacrificed by transcardial perfusion with $0.01 \mathrm{M}$ PBS, followed by $4 \%$ 
paraformaldehyde in PBS. The entire spinal cord was isolated from the vertebral column, fixed for $2 \mathrm{~h}$, soaked overnight in PBS, and cryoprotected in 30\% sucrose. Tissue blocks centered on the SCI epicenter (3-mm blocks for transectional sections) were frozen on dry ice and sectioned at the transectional plane $(10-\mu \mathrm{m}$ thickness for transectional slices). Bovine serum albumin (BSA) was used to block nonspecific binding, and the sections were incubated with anti-SIRT2 monoclonal antibody (Cell Signaling Technology, MA, USA) according to the manufacturer's instructions at $4^{\circ} \mathrm{C}$ overnight and then with anti-rabbit secondary antibody (Vector, $\mathrm{CA}$, USA) followed by incubation in $\mathrm{ABC}$ ( $\mathrm{ABC}$ Kit, Vector) at $37^{\circ} \mathrm{C}$ for $30 \mathrm{~min}$. The sections were then visualized by reacting with diaminobenzidine (DAB; Vector).

\section{Quantitative real-time polymerase chain reaction ( $q R T$ - PCR)}

The detection of the SIRT family in the injured spinal cord was performed as follows. Total RNA was extracted from fresh tissue cryosections using QIAzol reagent (Qiagen, Duesseldorf, Germany) following the manufacturer's instructions. The RNA concentration was assessed by a Nanodrop-100 system (Thermo Fisher Scientific, MA, USA), and samples were denatured and resolved on a $1 \%$ agarose gel to confirm the RNA integrity. cDNA was synthesized using $1 \mu \mathrm{g}$ of RNA by a QuantiTect Reverse Transcription kit (Qiagen) following the manufacturer's instructions. RT-qPCR was carried out on a StepOnePlus Real-Time PCR System (Applied Biosystems) using SYBR ${ }^{\circledR}$ Green Real-time PCR Master Mix (TaKaRa, Tokyo, Japan). The PCR conditions were $95^{\circ} \mathrm{C}$ for $5 \mathrm{~min}$, followed by 40 cycles of $95^{\circ} \mathrm{C}$ for $15 \mathrm{~s}$ and $60^{\circ} \mathrm{C}$ for $30 \mathrm{~s}$. The sequences of the primers for PCR and the length of product size are listed in Table 1. The relative mRNA levels of each gene were normalized based on GAPDH. Relative fold differences were determined using the delta-delta CT method, calculated as $\left.2^{-(\mathrm{Ct}}{ }_{\text {Target gene }}^{-\mathrm{Ct}}{ }_{\text {GAPDH }}\right)$. All experiments were performed at least three separate times. Data were analyzed using GenEx software version 6.1 (http://www.biomcc.com/genex-software.html).

\section{Differentiation of EpSCs into neurons}

EpSCs were cocultured as described in the Transwell coculture method. On day 7, the phenotypic expression of $\beta$ III-tubulin or MAP-2 (Cell Signaling Technology, MA, USA) in the EpSCs was examined by immunofluorescence or Western blot analysis as described below.

\section{Immunofluorescence}

The culture medium was discarded, and the coverslips from each group were rinsed with PBS (pH 7.4) twice. EpSCs were fixed in $4 \%$ paraformaldehyde for $30 \mathrm{~min}$, washed in PBS 3 times, and permeabilized with $0.1 \%$ Triton X-100 in PBS for $30 \mathrm{~min}$ at room temperature. After being washed in PBS 3 times, the EpSCs were incubated in PBS-Tween-20 (PBST) with 1\% BSA for 60 min to block nonspecific binding of antibodies. Then, the EpSCs were incubated with primary antibody overnight at $4^{\circ} \mathrm{C}$. Anti-SIRT2 (Cell Signaling Technology) and anti- $\beta$ III-tubulin (Cell Signaling Technology) were used as the primary antibodies. The nuclei were stained with DAPI. After being washed with PBS 3 times, the cultures were incubated with fluorescent Alexa 488 or 568 secondary antibodies (Invitrogen, CA, USA) for $1 \mathrm{~h}$. Immunofluorescence staining was examined using a Nikon Eclipse TE 300 microscope.

\section{Construction and infection of lentivirus-SIRT2}

For SIRT2 overexpression, lentiviral vectors were constructed by amplifying the cDNA of SIRT2 by PCR using specific primers (forward primer: 5'-AAGCTCGAGGAAGCGGCCTCAACACGG-3'; reverse primer: 5'-GGCGGATCCGTGGTTCCTTCAAGTTCGCA-3') and subcloning into the pLVXIRES-puro lentiviral vector using the XhoI and BamHI restriction sites, yielding lentivirus-SIRT2. Viruses were concentrated by using PEG-it virus precipitation solution (System Biosciences, Mountain View, CA) and stored at $-80^{\circ} \mathrm{C}$. The negative plasmid was also packaged and used as a negative control, designated lentivirusvector. This plasmid theoretically has no effect on any gene.

For lentivirus infection, EpSCs were seeded in 6-well plates followed by $1 \mu \mathrm{l}$ of $1,000 \times$ polybrene, and then, the cells were infected

Table 1. Primer sequences for RT-PCR

\begin{tabular}{cllc}
\hline Gene & \multicolumn{1}{c}{ Forward primer $\mathbf{5}^{\prime} \mathbf{-} \mathbf{3}^{\prime}$} & \multicolumn{1}{c}{ Reverse primer $\mathbf{5}^{\prime} \mathbf{- 3} \mathbf{3}^{\mathbf{2}}$} & Product size \\
\hline SIRT1 & GCAACAGCATCTTGCCTGAT & GTGCTACTGGTCTCACTT & 250 \\
SIRT2 & CTTCCTGGGCATGATGAT & ACCCTGACTGGGCATCTAT & 188 \\
SIRT3 & CAGCAACCTTCAGCAGTA & CCGTGCATGTAGCTGTTA & 278 \\
SIRT4 & TGACGGAGCTCCACGGATGC & ACACGCCGGTGCACAAAGTCA & 240 \\
SIRT5 & GTCATCACCCAGAACATTGA & ACGTGAGGTCGCAGCAAGCC & 251 \\
SIRT6 & TCCCAAGTGTAAGACGCAGT & GTTGCAGGTTGACAATGACC & 305 \\
SIRT7 & CCCCGGACCGCCATCTCA & CTCCAGGCCCAGTTCATTCAT & 453 \\
GAPDH & ACCACAGTCCATGCCATCAC & TCCACCACCCTGTTGCTGTA & 452 \\
\hline
\end{tabular}


with lentivirus-SIRT2 or lentivirus-vector.

\section{Western blot analysis}

SIRT2 expression in EpSCs was detected using western blotting assays $24 \mathrm{~h}$ post coculture. Possible activation of the MEK1/2 signaling pathway in the EpSCs cocultured with macrophages was measured at 0,15 and $30 \mathrm{~min}$ postcoculture. In brief, the cells were lysed with lysis buffer (Sigma-Aldrich, MO, USA) containing phenylmethanesulfonyl fluoride (PMSF). The lysates were centrifuged at $12,000 \mathrm{~g}$ at $4^{\circ} \mathrm{C}$ for $10 \mathrm{~min}$, and the supernatant was collected and preserved at $-80^{\circ} \mathrm{C}$ for later use. The protein concentrations were determined using a BCA Protein Assay Kit (Pierce, IL, USA). The proteins were separated by $15 \%$ sodium dodecyl sulfate polyacrylamide gel electrophoresis (SDS-PAGE) and then transferred to polyvinylidene difluoride (PVDF) membranes using a standard protocol. The membranes were blocked by incubation for $1 \mathrm{~h}$ at room temperature in 5\% fat-free dry milk in Tris-buffered saline containing $0.1 \%$ Tween 20 (TBS-T). The membranes were incubated with primary antibodies diluted 1:1000 in TBST containing 5\% BSA and probed with the appropriate secondary antibodies. The membranes were exposed and scanned using an Odyssey ${ }^{\circledR}$ Infrared Imaging System.

To elucidate the signaling pathway involved in SIRT2-mediated EpSC proliferation, we treated the EpSCs with a TrkB antagonist (ANA12, Selleckchem, TX, USA) or a MEK1/2 inhibitor (PD0325901, Cell Signaling Technology) for $2 \mathrm{~h}$. Then, the EpSCs were cocultured with macrophages as described above, and western blot analysis was performed.

For analysis of the spinal cord, the samples were prepared as follows. After RT-PCR, the remaining sample was centrifuged at 1,500 rpm for $30 \mathrm{~min}$; the supernatant (crude protein) was collected. Determination of the protein concentration was performed by the Bradford method. Then, Western blot was performed as described above.

The following antibodies were used: MAP-2 (Abcam, MA, USA), acetylated $\alpha$-tubulin (AC $\alpha$-tubulin) (Proteintech, IL, USA), a-tubulin (Proteintech), p-TrkB Y816 (Novus Biologicals, CO, USA), TrkB, SIRT2, p-MEK, p-ERK1/2 and $\beta$-actin (all from Cell Signaling Technology). An image analytic system was used to detect the gray degree values of the positive signals.

\section{Statistical analysis}

Data are presented as the mean \pm SD and were analyzed by GraphPad Prism V 5.00 for Windows (GraphPad Software, La Jolla, USA). Statistical significance was determined by Student's ttest or ANOVA followed by Newman-Keuls post hoc test. p-values less than 0.05 were considered statistically significant.

\section{RESULTS}

\section{Expression of SIRT2 in the ependymal region after spinal cord injury}

WT mice underwent laminectomy and spinal cord contusion at the T8-9 segment. As the first week post SCI is usually referred to as the active period of the M2 reaction, the mRNA level of the SIRT family was measured on day 3 with RT-PCR. SIRT2 showed the highest level among all the family members, indicating its important role in this process (Fig. 1a). Then, the expression of SIRT2 at the SCI site was detected by immunohistochemistry and western blot assays. The spinal cord samples centered at the injury site were isolated on days 1, 3 and 7 after injury. On day 1 after injury, positive SIRT2 immunostaining could be observed at the injury site, which was located in both the ependymal area and the gray matter of the spinal cord, presenting cytoplasmic staining. The cells with positive staining in the gray matter were preliminarily identified as neurons according to cell morphology. Weakly positive staining for SIRT2 was observed in the ependymal zone on day 1 post injury in the WT mice with SCI, while strong SIRT2 positive staining was observed on day 3 post injury, especially in the ependymal region (Fig. 1b), which was still positive on day 7 . In contrast, only weak staining of SIRT2 could be detected in the central canal of the mice in the sham group. Western blot assays obtained similar results (Fig. 1c and 1d).

\section{M2 coculture upregulated the expression of SIRT2 in the EpSCs in vitro}

To explore the possible role of SIRT2 during EpSC differentiation, we cocultured EpSCs with M0, M1 or M2 macrophages. RTPCR was performed on day three to assess the SIRT2 mRNA levels in the EpSCs of different groups. The expression of SIRT2 mRNA was significantly higher in the EpSCs cocultured with M2 than in the other EpSCs ( $<<0.001)$ (Fig. 2a). In addition, semiquantitative analysis with western blotting showed that SIRT2 expression was significantly increased in the M2 coculture group ( $\mathrm{p}<0.001)$ (Fig. $2 \mathrm{~b})$. Consistent with our previous results, the EpSCs in the M2 coculture group showed improved growth, including cell body enlargement and neurite elongation, on day 7 post coculture. The EpSCs showed positive $\beta$ III-tubulin staining, indicating differentiation towards neurons. Interestingly, the EpSCs cocultured with M2 demonstrated significant SIRT2 immunostaining on the third day of coculture, which was localized in the cytoplasm and could be observed throughout the experiment. Weakly positive SIRT2 staining could be found in the M0 group, and the staining was even weaker in the $\mathrm{M} 1$ group (Fig. 2c).

Since M2 could affect the expression of SIRT2 in EpSCs, the tem- 
a

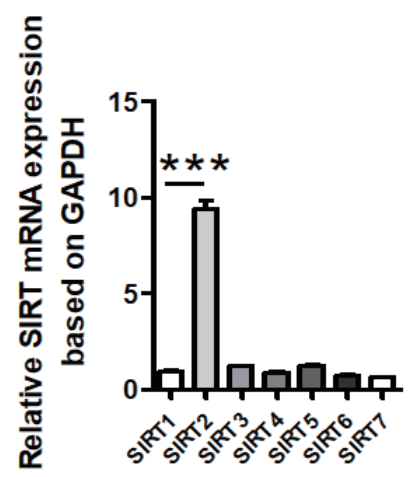

C

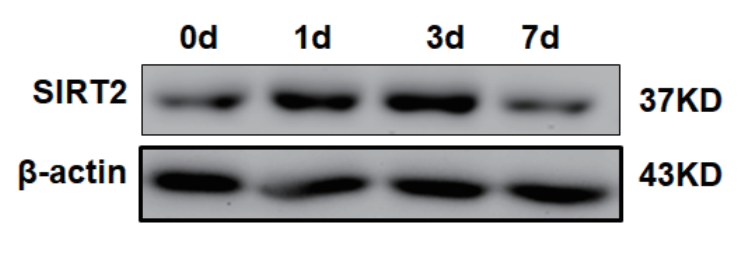

d

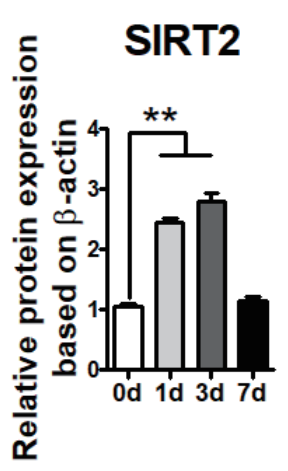

b

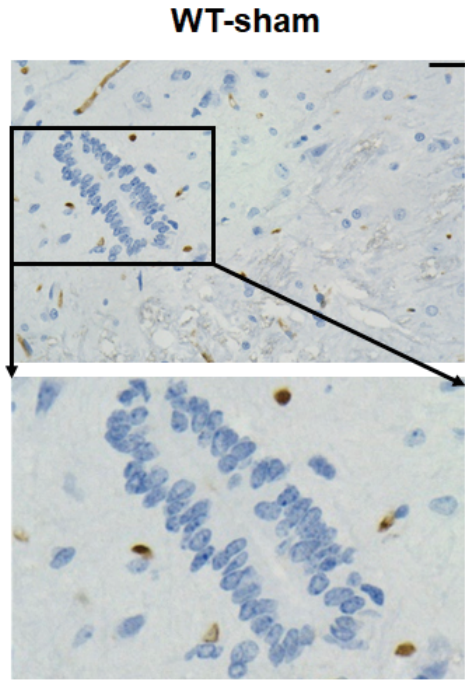

BDNF $^{--} \mathrm{SCl}$

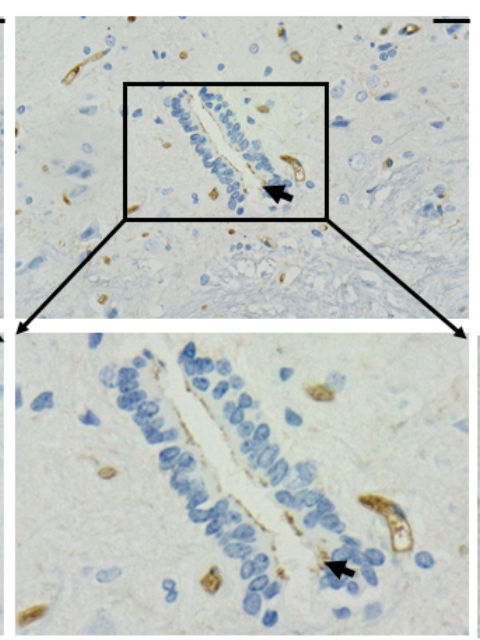

WT-SCI

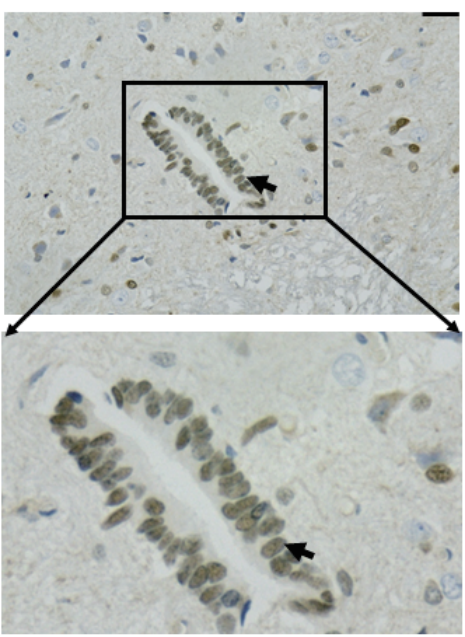

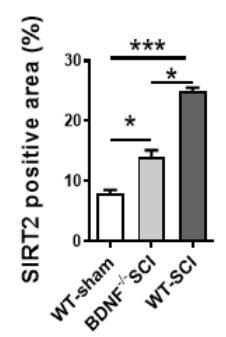

Fig. 1. SIRT2 expression in the injured spinal cord on day 3 post SCI. (a) Spinal cord tissue was isolated from the WT mice with SCI on day 3 after SCI, and RT-PCR was performed to examine the expression of the SIRT family. SIRT2 had the highest expression level compared with other members in the spinal cord. (b) Representative IHC images of SIRT2 on day 3 after SCI in the central canal of the spinal cord $(\times 200)$. Scale bars: $50 \mu \mathrm{m}$. The arrows indicate the positively stained cell in the central canal of the spinal cord. WT-sham: WT mice undergoing laminectomy only; WT-SCI: WT mice with $S C I$. BDNF $F^{\prime}$-SCI: BDNF KO mice with SCI. A summary of the semiquantitative analysis of the SIRT2-positive area is shown. (c) Western blotting was performed to examine the temporal expression of SIRT2 after SCI. Statistical analysis is shown in (d). Data represent three independent experiments. ${ }^{*} \mathrm{p}<0.05,{ }^{* *} \mathrm{p}<0.01,{ }^{* * *} \mathrm{p}<0.001$.

poral change in SIRT2 expression in the EpSCs cocultured with M2 was also studied using RT-PCR and western blot analyses. As shown in Fig. 2d, there was a low level of SIRT2 expression in normal EpSCs before coculture. The expression of SIRT2 increased at $6 \mathrm{~h}$ after coculture, peaking at $48 \mathrm{~h}$ and then declining at $72 \mathrm{~h}$ post coculture. Western blot analysis obtained a similar result (Fig. 2e).

\section{SIRT2 played an important role in the EpSC differentiation mediated by the M2 coculture}

M2 conditioned medium facilitates the differentiation of EpSCs into neurons. It also upregulates the expression of SIRT2 in cocultured EpSCs. Therefore, does M2 promote EpSC differentiation towards neurons through its effect on SIRT2 expression? To address this question, we cocultured the EpSCs from different groups with M2 macrophages. To obtain EpSCs overexpressing SIRT2, we successfully constructed plasmid lentivirus-SIRT2 and infected EpSCs (Fig. 3a and 3b). WT EpSCs demonstrated positive staining for $\beta$ III-tubulin and SIRT2, together with robust cellular growth, on the $7^{\text {th }}$ day post coculture (Fig. 2c). A similar effect was observed in the SIRT2 overexpression group without M2 coculture. However, neither SIRT2 nor $\beta$ III-tubulin immunostaining could be observed in the EpSCs derived from the SIRT2 ${ }^{-}$mice at the same time (Fig. 3c). Decreased SIRT2 and ßIII-tubulin immunostaining was also observed in the EpSCs preincubated with ANA12, 
a

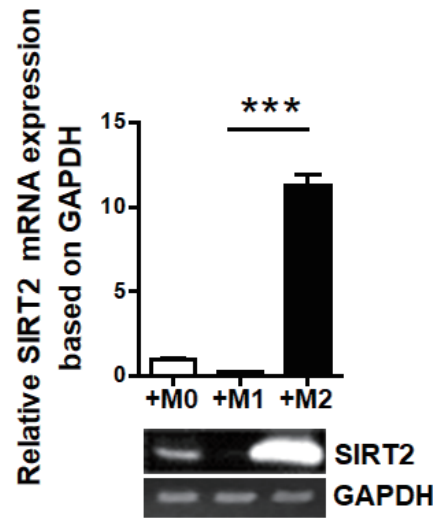

b

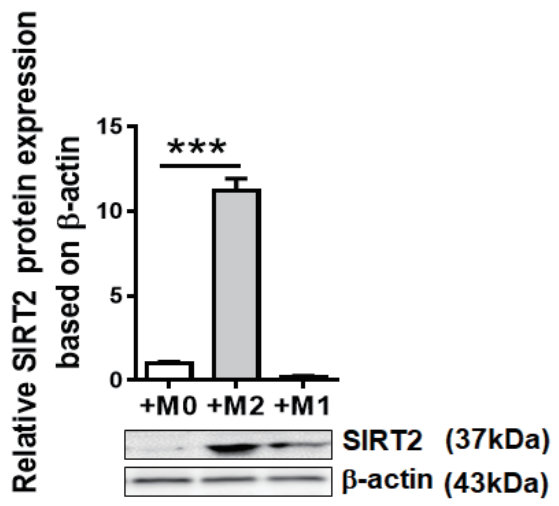

\section{SIRT2 $\quad \beta$ Ill-tublin $\quad$ DAPI $\quad$ Merge}

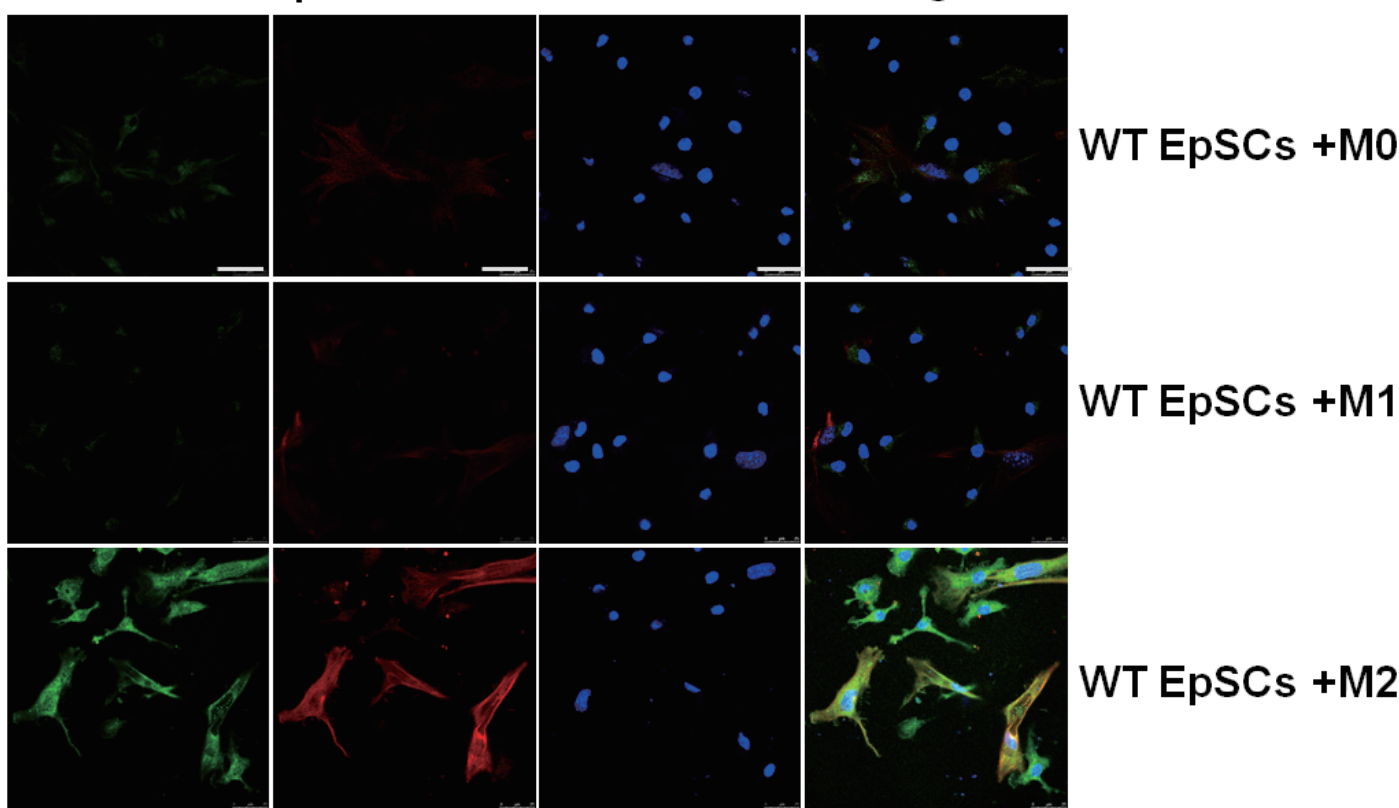

d

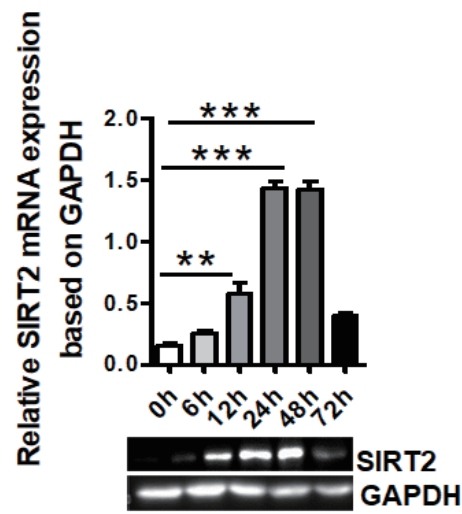

e

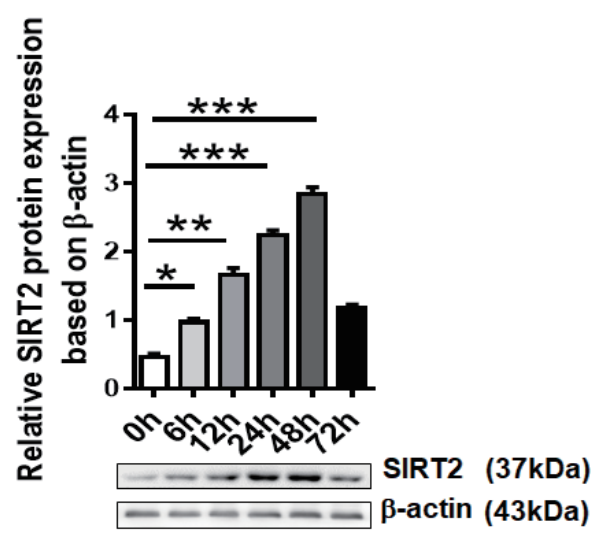

Fig. 2. M2 promoted EpSC differentiation by upregulating SIRT2 expression. SIRT2 expression in the EpSCs cocultured with M1, M2 or M0 was detected by RT-PCR (a) and western blots (b). SIRT2 and $\beta$ III-tubulin expression in the EpSCs from the WT mice cocultured with M1, M2 or M0 was detected by immunofluorescence (c). (Blue: DAPI; green: SIRT2; red: $\beta$ III-tubulin; $\times 400$ ). Scale bars: $25 \mu \mathrm{m}$. RT-PCR (d) and western blotting assays (e) were used to detect temporal SIRT2 expression in the EpSCs cocultured with M2 macrophages. Data represent three independent experiments. ${ }^{*} \mathrm{p}<0.05$, ${ }^{* *} \mathrm{p}<0.01,{ }^{* * *} \mathrm{p}<0.001$ 
a

Lane M: DNA ladder

Lane 1: lentivirus-vector

Lane 2: lentivirus-SIRT2

\section{12}

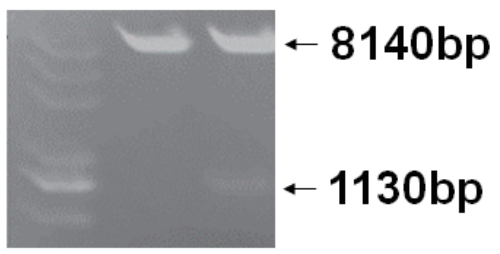

b

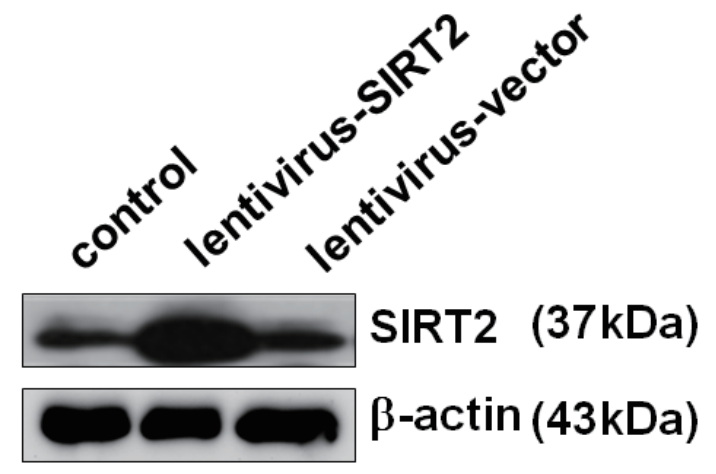

c SIRT2 $\quad \beta$ III-tubulin $\quad$ DAPI $\quad$ Merge

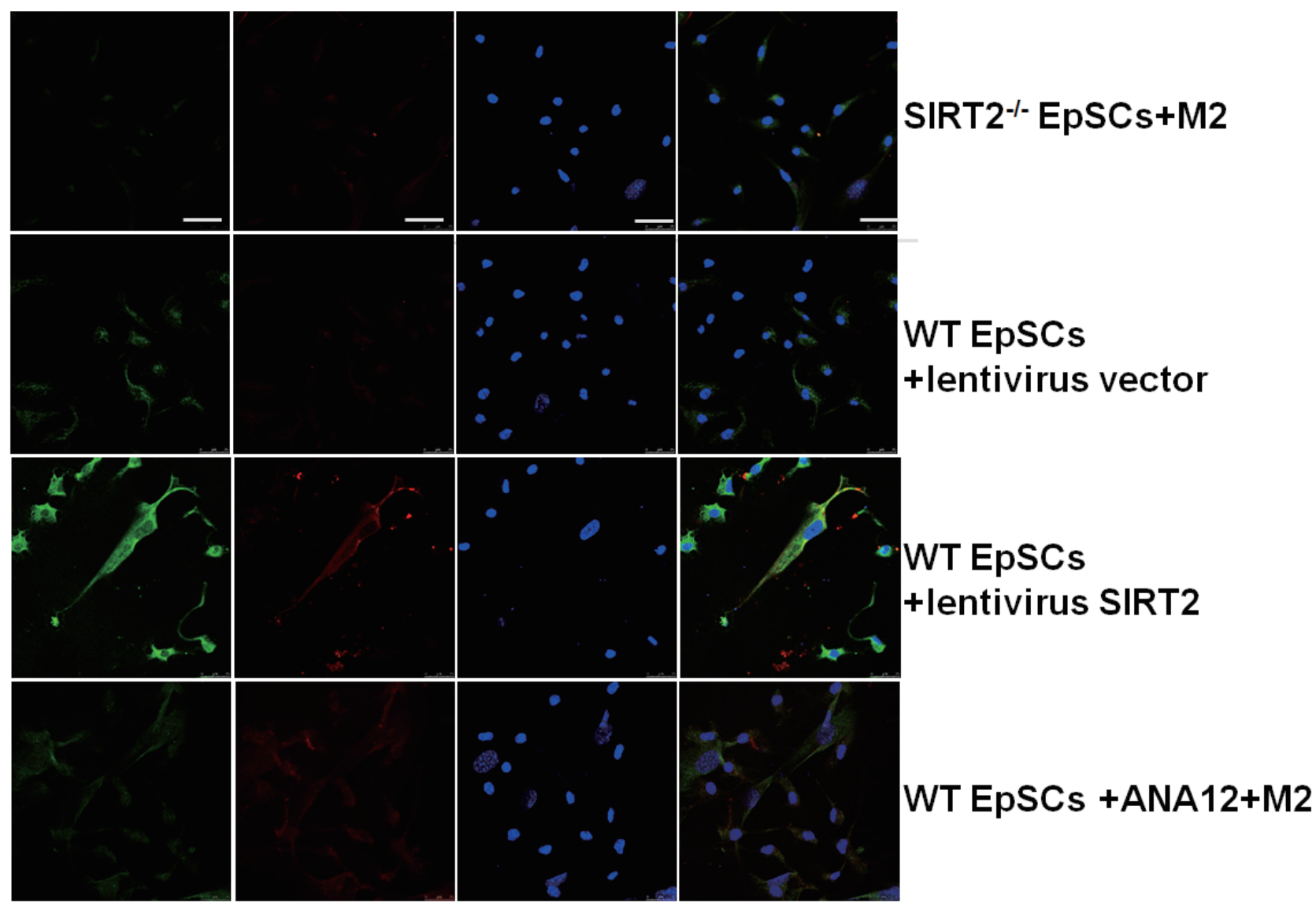

Fig. 3. SIRT2 is critical for the differentiation of EpSCs. (a) Construction and identification of the lentivirus-SIRT2 plasmid. The lentivirus-SIRT2 plasmid was digested with XhoI and BamHI. Lane 1, lentivirus-vector; lane 2, lentivirus-SIRT2. (b) EpSCs were infected with lentivirus-SIRT2 or lentivirusvector. SIRT2 expression was identified by western blots. (c) EpSCs from the SIRT2 ${ }^{-/}$mice or EpSCs from the WT mice pretreated with TrkB inhibitor (ANA12) were cocultured with M2 macrophages. EpSCs from the WT mice were infected with lentivirus-SIRT2 or lentivirus-vector for 48 h. SIRT2 and $\beta$ III-tubulin levels were then detected by immunofluorescence. (Blue: DAPI; green: SIRT2; red: $\beta$ III-tubulin; $\times 400$ ). Scale bars: $25 \mu \mathrm{m}$. 
a selective inhibitor of TrkB. The levels of SIRT2 significantly correlated with the differentiation status of the EpSCs, suggesting a key role for SIRT2 in guiding EpSC differentiation.

\section{SIRT2 promoted EpSC differentiation by its a-tubulin deacetylase activity}

The mechanism by which SIRT2 regulates EpSC differentiation remains elusive. It was reported that acetylated $\alpha$-tubulin inhibits axon and neurite outgrowth from neurons. We examined whether SIRT2 regulated neuronal differentiation through its a-tubulin deacetylase activity. EpSCs were cocultured with M0, M1 or M2 for $24 \mathrm{~h}$, and then, the expression of MAP-2 and Ac $a$-tubulin was measured using western blots (Fig. 4a). Similar to the immunofluorescence results, MAP-2 expression in the EpSCs was significantly upregulated in the M2 coculture group, while that of Ac a-tubulin was significantly decreased. No significant change in Ac a-tubulin expression was found in the M1 or M0 coculture group.
These results confirmed that M2 coculture could deacetylate Ac a-tubulin in the EpSCs and thus promote the differentiation of EpSCs into neurons. We further investigated whether the reduction of Ac a-tubulin was realized through the SIRT2 pathway. In the WT EpSC group, the Ac a-tubulin expression levels gradually decreased from 24 to $48 \mathrm{~h}$ during coculture with M2 and then returned to baseline at $72 \mathrm{~h}$ (Fig. $4 \mathrm{~b}$ and $4 \mathrm{c}$ ). There was no apparent fluctuation in the Ac a-tubulin expression in the EpSCs derived from the SIRT2 ${ }^{-/}$mice (Fig. $4 \mathrm{~b}$ and $4 \mathrm{c}$ ).

\section{M2 upregulated SIRT2 expression in the EpSCs through the BDNF-TrkB signaling pathway}

M2 can upregulate the expression of SIRT2 in cocultured EpSCs, and we determined the possible mechanism involved in the present study. BDNF can increase neural differentiation and relieve the inflammatory response. SIRT2 immunostaining in the sections from the $\mathrm{BDNF}^{-/}$mice was significantly lower than that of the WT a

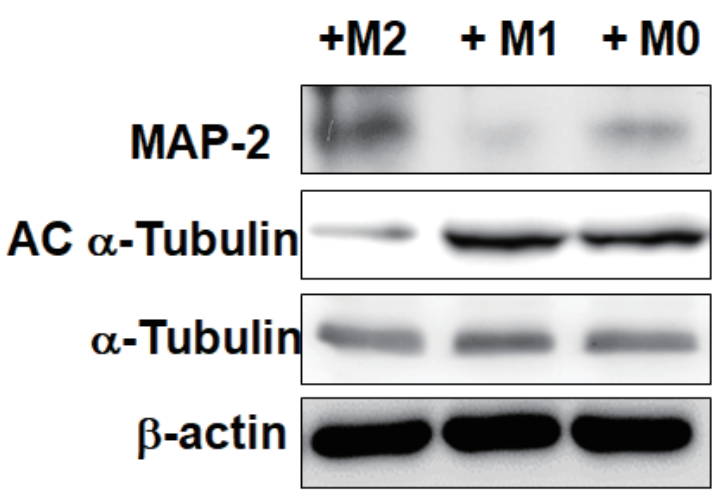

$\mathrm{kDa}$

200

55

55

43

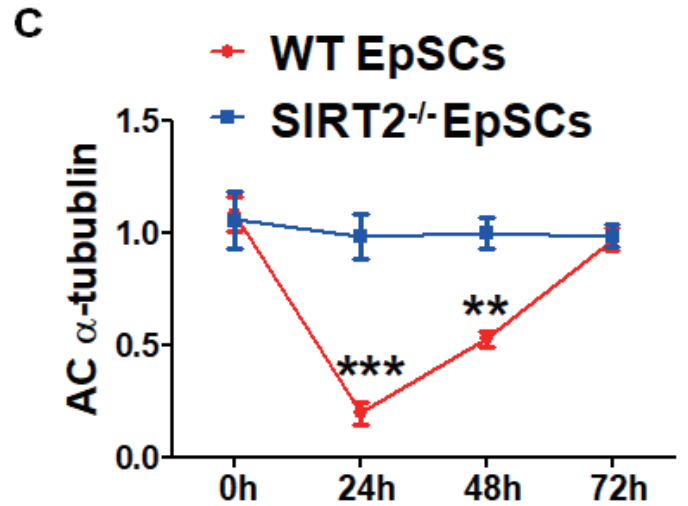

SIRT2-I-EpSCs

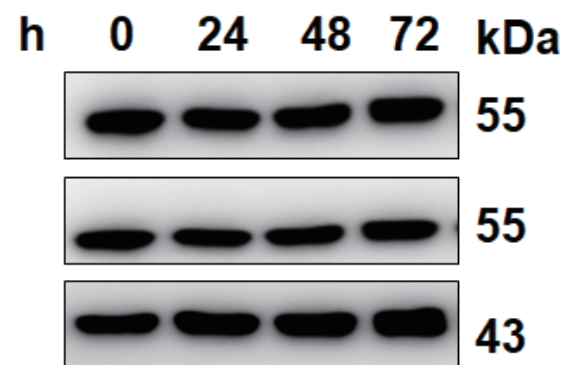

Fig. 4. Changes in acetylated $a$-tubulin expression of the EpSCs during coculture with macrophages. (a) Detection of the expression of MAP-2 and acetylated $\alpha$-tubulin (Ac $\alpha$-tubulin) in the EpSCs after coculture with M0, M1 or M2 for 24 h. Significantly decreased Ac $\alpha$-tubulin and increased MAP2 were observed in the EpSCs cocultured with M2. (b) Detection of the temporal expression of Ac a-tubulin and a-tubulin in differently derived EpSCs when cocultured with M2 (0, 6, 12,24 h) by western blotting assays. The Ac $\alpha$-tubulin expression levels in the EpSCs derived from the WT mice gradually decreased from 24 to $48 \mathrm{~h}$ upon coculture with M2 and then returned to baseline at $72 \mathrm{~h}$; in contrast, there were no apparent changes in the Ac a-tubulin expression in the EpSCs derived from the SIRT2 ${ }^{-*}$ mice. Statistical analysis is shown in (c). Data represent three independent experiments. ${ }^{* *} \mathrm{p}<0.01$, ${ }^{* * *} \mathrm{p}<0.001$. 

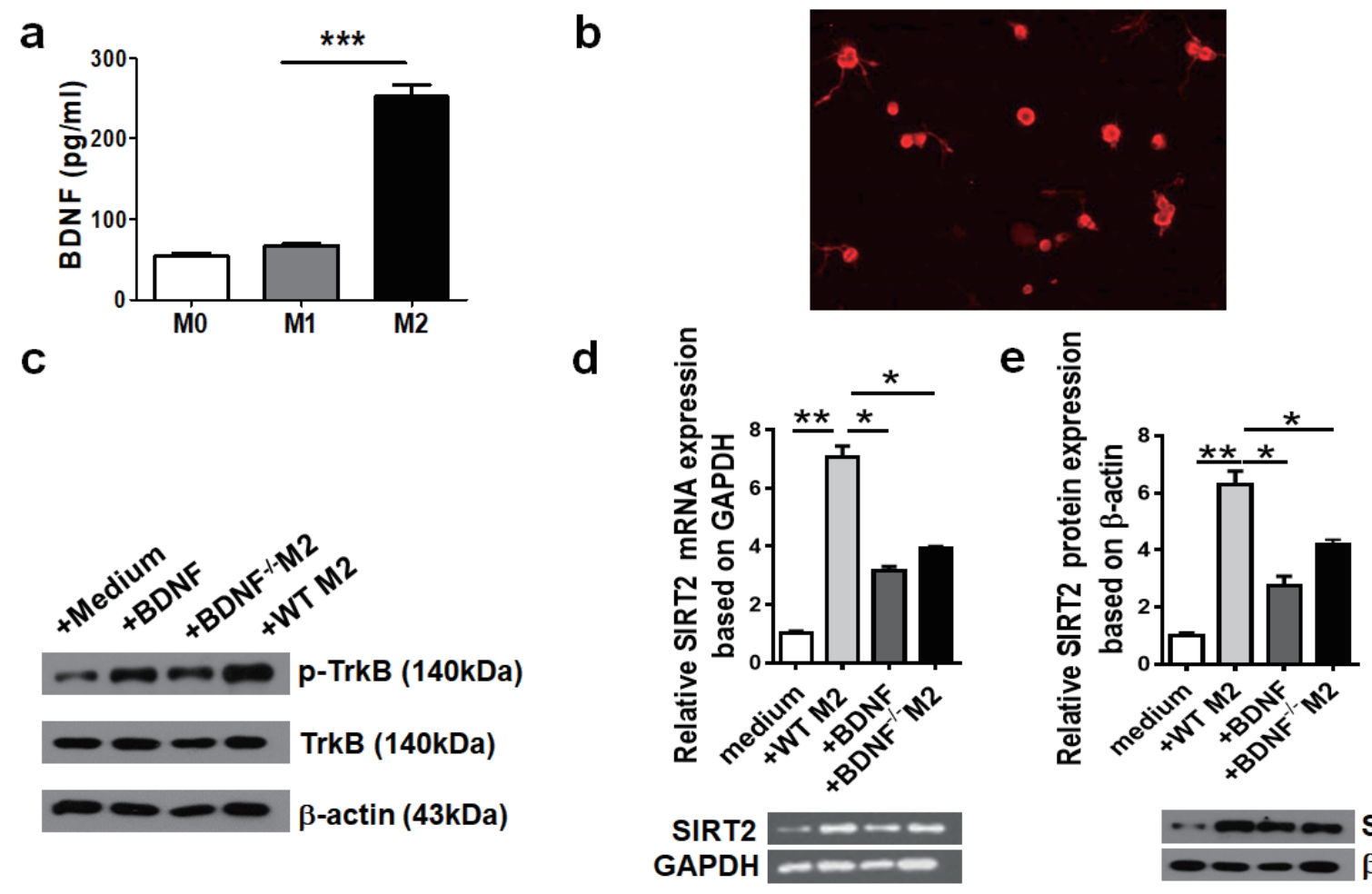

SIRT2 (37kDa) $\beta$-actin (43kDa)

Fig. 5. M2 upregulated SIRT2 expression in the EpSCs through BDNF-TrkB signaling. (a) The secretion of BDNF in different macrophage conditioned media was detected by ELISAs. The BDNF protein in the M2 conditioned medium was significantly higher than that in the M1 or M0 conditioned medium. (b) The expression of TrkB receptor in the EpSCs was observed by immunofluorescence staining, which was mainly located on the surface of cell bodies, axons and dendrites. (c) Representative immunoreactive bands of $\mathrm{p}-\operatorname{TrkB}$ and TrkB in the EpSCs cocultured with M2 derived from the WT or $\mathrm{BDNF}^{-/}$mice. The $\mathrm{p}$-TrkB expression in the EpSCs was significantly induced when cocultured with M2 from the WT mice but had no change when cocultured with M2 from the $\mathrm{BDNF}^{/}$mice. RT-PCR (d) and western blot assays (e) were used to detect SIRT2 expression in the EpSCs of different groups. The EpSCs cocultured with M2 from the $\mathrm{BDNF}^{-/}$mice showed decreased SIRT2 expression. Data represent three independent experiments. ${ }^{*} \mathrm{p}<0.05$, ${ }^{* *} \mathrm{p}<0.01,{ }^{* * *} \mathrm{p}<0.001$.

mice (Fig. 1b); thus, we explored whether M2 upregulated SIRT2 expression in EpSCs through the BDNF/TrkB signaling pathway. First, we detected the BDNF protein expression in the M2 conditioned medium by ELISAs (Fig. 5a) and found that its level was significantly higher than that of the M1 and M0 groups. Second, we observed the expression of the TrkB receptor in EpSCs by immunofluorescence (Fig. 5b) and found positive staining mainly located on the surface of cell bodies, axons and dendrites. Western blot analysis showed that the level of p-TrkB in EpSCs was significantly elevated following coculture with M2 from the WT mice for $6 \mathrm{~h}$ (Fig. 5c). Positive staining for $\beta I I I-t u b u l i n$ and SIRT2 decreased due to pretreatment with an inhibitor of TrkB (ANA12) (Fig. 3c). The expression of intracellular phosphorylated TrkB, however, was not as evident if the EpSCs were cocultured with M2 derived from the $\mathrm{BDNF}^{-1}$ mice but not the WT mice, suggesting that BDNF is an important factor in activating TrkB on the surface of the EpSCs. More importantly, both RT-PCR (Fig. 5d) and western blot analyses (Fig. 5e) confirmed that there was a certain amount of SIRT2 expression in the EpSCs cocultured with M2 from the WT mice, while the expression of SIRT2 was significantly reduced if the M2 macrophages were derived from the $\mathrm{BDNF}^{-}$mice.

\section{MEK1/2-ERK1/2 activation resulting from phosphoryla- tion of TrkB upregulated SIRT2 expression in the EpSCs}

To explore the downstream signals of the BDNF/TrkB pathway, we cocultured EpSCs with M2 macrophages, and the effect of different pretreatments on SIRT2 expression was studied. ERK1/2 regulates SIRT2 deacetylase activity, and the phosphorylation of ERK1/2 depends on the activation of MAPK kinase (MEK) $1 / 2$. In the present study, we attempted to determine whether BDNF/ TrkB affected the activation of MEK-ERK1/2 and thus regulated the expression of SIRT2 (Fig. 6). First, EpSCs were pretreated with ANA-12, a potent and selective TrkB antagonist, for $2 \mathrm{~h}$ followed by coculture with M2. Compared with the control group, in which the EpSCs were directly cocultured with M2, the ANA-12 group showed substantially decreased $\mathrm{p}$-TrkB levels. The levels of $\mathrm{p}$ - 
a

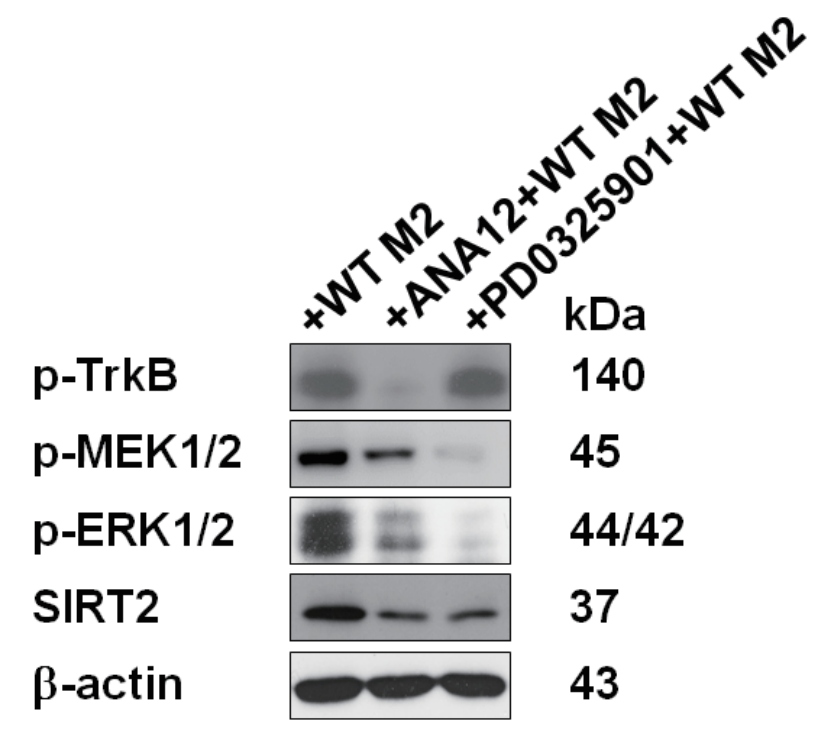

b

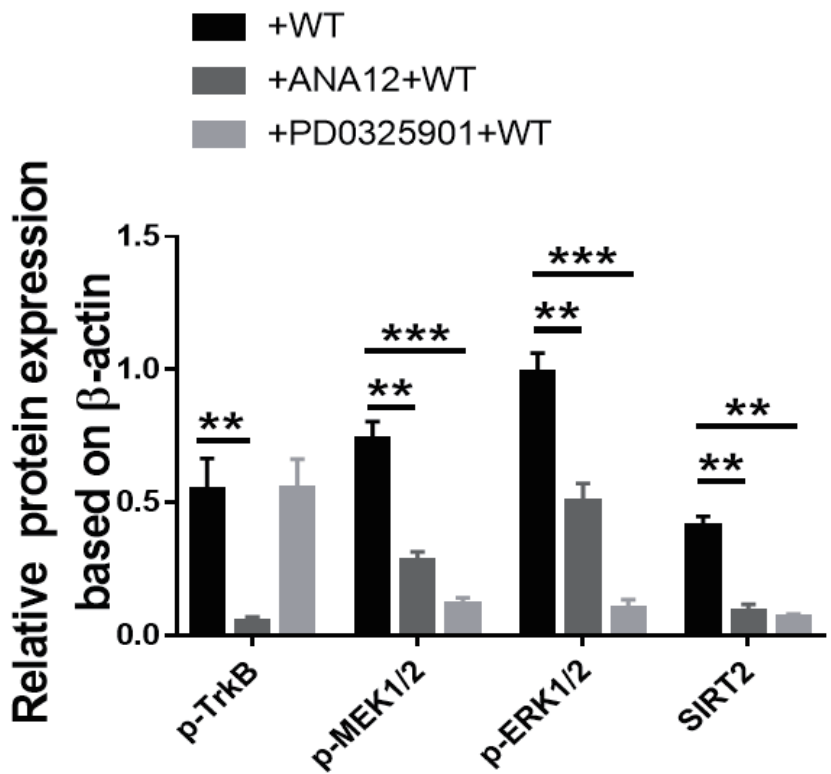

Fig. 6. The MEK1/2 signaling pathway was activated in the SIRT2-expressing cells. EpSCs were pretreated with a TrkB antagonist (ANA-12) or MEK1/2 inhibitor (PD0325901) for $2 \mathrm{~h}$ and then cocultured with M2 from the WT mice. The levels of p-TrkB, p-MEK1/2, p-ERK1/2, SIRT2 and $\beta$-actin were detected by western blot analysis.

MEK1/2 and p-ERK1/2 also decreased, and the expression of SIRT2 was obviously downregulated. In another group, the EpSCs were pretreated with PD0325901, a selective, non-ATP competitive MEK inhibitor. The subsequent coculture with M2 did not change the level of $\mathrm{p}$-TrkB. However, compared with those of the control group, the levels of $\mathrm{p}$-MEK1/2 and p-ERK1/2 as well as the expression of SIRT2 were significantly reduced. The above results suggested that MEK1/2-ERK1/2 acted downstream of BDNKTrkB. BDNF-TrkB regulated SIRT2 expression in EpSCs via the MEK1/2-ERK1/2 signaling pathway.

\section{DISCUSSION}

Transplantation of various types of stem cells, such as neural stem cells (NSCs), bone marrow mesenchymal stem cells (MSCs) and induced pluripotent stem cells (iPSCs), to rescue the injured spinal cord has recently attracted increasing attention [14-16]. Neural stem/progenitor cells (NSPCs) in the adult rat spinal cord show self-renewal but no tumorigenic potential. Transplantation of NSPCs in rodents with SCI has been shown to increase tissue retention, secrete beneficial nutrient factors, and improve functional recovery [17]. Alfaro-Cervello et al. [18] explored the source of spinal cord-derived NSPCs and confirmed that these cells were ependymal cells with two long cilia, which showed a Vimentin ${ }^{+}$, $\mathrm{CD}_{24}{ }^{+}$, Fox $1^{+}$, Sox $^{+}, \mathrm{CD}_{133^{+}}$, nestin ${ }^{-}$and GFAP phenotype. Spi- nal cord NSPCs are also known as ependymal stem cells (EpSCs) because they maintain a resting state in an intact spinal cord and participate in forming the central spinal canal while rapidly proliferating and migrating to the injured site after SCI. Pfenninger [19] isolated $\mathrm{CD} 133^{+} / \mathrm{CD} 24^{+}$EpSCs from mouse spinal cord tissues using immunomagnetic beads. EpSCs differentiate into motor neurons in vitro with HB9 immunostaining [20]; however, they mainly differentiate into astrocytes in vivo and contribute to the glial scar formed 1 week after injury [21], which protects the nerve tissue from further damage and hinders the repair of damage (acting as a mechanical and chemical barrier for axon elongation).

Numerous studies in multiple laboratories around the world have explored the mechanism that regulates the biological behavior of EpSCs in vivo, especially their growth and differentiation. The inhibitory microenvironment formed following SCI is considered to play an important role in this process. Secondary inflammation, mainly induced by activated macrophages, is one of the most important events in the microenvironment of the injured spinal cord, and this process has recently attracted increasing attention [22, 23]. Activated microglia/macrophages have either detrimental or beneficial effects on neural regeneration based on different subsets. The proinflammatory M1 cell response, which is rapidly induced and sustained after SCI, contributes to protracted cell and tissue loss, whereas the alternative anti-inflammatory M2 type, which is induced transiently, is believed to promote cell pro- 
tection, regeneration and plasticity. Our previous study found that M2 conditioned medium favored EpSC differentiation towards neurons, but the underlying molecular mechanism remains elusive [9]. If the mechanism by which M2 regulates EpSC differentiation can be illuminated and a scheme to promote EpSC differentiation into neurons can be designed on this basis, it will provide a promising strategy for the recovery from SCI.

Sirtuins (SIRTs) are NAD ${ }^{+}$-dependent class III histone deacetylases (HDACs). As one of the seven SIRT homologues, SIRT2 catalyzes various biological processes, such as metabolism and gene expression [24]. SIRT2 is involved in the regulation of the cell cycle, oxidative stress response and microtubule dynamics [25]. We observed the expression of SIRT2 in the injury site after SCI through immunohistochemical staining. Semiquantitative analysis using western blots showed that SIRT2 expression was significantly higher in the spinal cord of the mice with SCI than in the mice that underwent sham operation only, suggesting that SIRT2 plays a role in the pathogenesis and prognosis of SCI. The present study aimed to explore in vitro whether M2 can regulate the expression of SIRT2 in EpSCs and thereby affect their growth and differentiation. M2 coculture facilitated the differentiation of EpSCs into neurons with positive $\beta$ III-tubulin staining. Furthermore, compared with M1 or M0, M2 coculture could upregulate the expression of SIRT2 in EpSCs. More interestingly, for the EpSCs derived from the SIRT2 ${ }^{-1}$ mice, M2 did not promote their differentiation towards neurons, confirming that the effect of M2 on the differentiation of EpSCs was at least partially mediated by SIRT2.

We further studied the mechanism by which M2 induced neuronal differentiation through SIRT2. Microtubules (MTs) are complex structures consisting of asymmetric heterodimers of $a$ - and $\beta$-tubulin. As a major component of MTs, a-tubulin contributes to MT modification via acetylation/deacetylation at lysine 40 (K40) [26]. MT dynamics have profound functional effects on cellular transport, migration, differentiation, and division [27]. A previous study reported that acetylated $\alpha$-tubulin (Ac- $\alpha$-tubulin) is generally found in stable MT polymers and inhibits axon and neurite outgrowth from neurons [28]. It has also been reported that the knockout of SIRT2 expression can inhibit the differentiation of midbrain neurons into dopaminergic neurons in embryonic mice [29]. Based on these findings, in the present study, we examined whether SIRT2 regulates neuronal differentiation through its a-tubulin deacetylase activity. Consistent with these reports, our findings demonstrated that the intracellular level of Ac a-tubulin was significantly decreased when EpSCs were cocultured with WT M2, suggesting that SIRT2 upregulation by M2 coculture deacetylated stable Ac- $\boldsymbol{\alpha}$-tubulin in the EpSCs. This change in microtubule dynamics contributes to the differentiation of EpSCs into neurons, manifested as increased MAP-2 levels. After coculture with M2 macrophages, no significant change in the Ac a-tubulin levels was detected in the EpSCs from the SIRT $2^{-/}$mice, further indicating that the effect of M2 macrophages on EpSC differentiation was achieved by SIRT2 expression.

Another interesting question is how M2 regulates the expression of SIRT2 in EpSCs. Since this noncontact coculture was carried out in transwell chambers, the effect of M2 is likely achieved through paracrine mechanisms. BDNF, one of the key cytokines secreted by $\mathrm{M} 2$ macrophages, can induce neural differentiation in stem cells and has the potential to repair the nervous system [30, 31]. Once bound to its specific receptor, BDNF and the tyrosine kinase receptor TrkB increase the branching of cortical and hippocampal neurons in dissociated cultures and organotypic slices [32]. We attempted to confirm whether BDNF had an impact on the expression of SIRT2. The expression of BDNF in M2 conditioned medium was detected, and the expression of TrkB on the surface of EpSCs was observed, suggesting that M2 can influence the biological behavior of EpSCs through the BDNF/TrkB signaling pathway. As expected, further experiments showed a relationship between the phosphorylation level of TrkB in EpSCs and BDNF in the medium. Our results indicated that BDNF secreted by M2 can activate TrkB on the surface of EpSCs, thus inducing the expression of SIRT2. To our knowledge, this is the first study to show that BDNF/TrkB upregulates the expression of SIRT2.

We further explored the potential molecules involved in the BDNF-TrkB-SIRT2 signaling pathway. After binding with BDNF, TrkB dimerizes and undergoes autophosphorylation at specific tyrosine residues of the intracellular domain [33]. These phosphotyrosines are docking sites for adaptor proteins that lead to the activation of several signaling cascades, including mitogenactivated protein kinases (MAPKs) [34], phospholipase C-gamma (PLC $\gamma$ ) [35] and phosphoinositide-3-kinase (PI3K) [36]. It has been reported that activation of MAPK kinase (MEK) $1 / 2$ leads to the phosphorylation of ERK1/2 via the Thr-Glu-Tyr recognition motif, and the latter regulates SIRT2 deacetylase activity [13]. In the present study, we found that ANA, an antagonist of TrkB, could not only downregulate the level of p-TrkB in EpSCs but also reduce the levels of $\mathrm{p}$-MEK1/2 and p-ERK1/2, and the expression of SIRT2 was also significantly downregulated. In contrast, an MEK inhibitor (PD0325901) had no influence on the level of p-TrkB, while it dramatically decreased the expression levels of $\mathrm{p}$-ERK1/2 and SIRT2. The results suggested that phosphorylation of TrkB induced by BDNF resulted in the phosphorylation of ERK1/2 followed by the upregulation of SIRT2. Taken together, our study suggests that M2 regulates SIRT2 expression in EpSCs via the BDNF/TrkB-MEK/ERK- SIRT2 signaling pathway. 
We confirmed in vitro that the M2-type macrophage response can promote the differentiation of EpSCs into neurons at least partially through upregulating the expression of SIRT2. These results provide a theoretical basis and a new strategy for improving the recovery from spinal cord injury, such as regulating the survival, growth and differentiation of EpSCs by increasing the proportion of M2 cells in the local microenvironment of SCI or upregulating the expression of SIRT2 in EpSCs. There may be other factors and signaling pathways besides BDNF involved in the regulation of SIRT2 by M2, which needs further study.

\section{ACKNOWLEDGEMENTS}

This work was supported by: WJ2015MB087, Health and family planning commission of Hubei province; 2014CFB205, Natural science and technology foundation of Hubei province; 81501377, National Natural Science Foundation of China; RMYD2018M53, Guide fund of Renmin Hospital of Wuhan University.

\section{CONFLICT OF INTEREST}

We, all authors of the paper, declare no conflict of interest.

\section{REFERENCES}

1. Park HW, Oh S, Lee KH, Lee BH, Chang MS (2018) Olig2expressing mesenchymal stem cells enhance functional recovery after contusive spinal cord injury. Int J Stem Cells 11:177-186.

2. Piltti KM, Funes GM, Avakian SN, Salibian AA, Huang KI, Carta K, Kamei N, Flanagan LA, Monuki ES, Uchida N, Cummings BJ, Anderson AJ (2017) Increasing human neural stem cell transplantation dose alters oligodendroglial and neuronal differentiation after spinal cord injury. Stem Cell Reports 8:1534-1548.

3. Sankavaram SR, Hakim R, Covacu R, Frostell A, Neumann S, Svensson M, Brundin L (2019) Adult neural progenitor cells transplanted into spinal cord injury differentiate into oligodendrocytes, enhance myelination, and contribute to recovery. Stem Cell Reports 12:950-966.

4. Cawsey T, Duflou J, Weickert CS, Gorrie CA (2015) Nestinpositive ependymal cells are increased in the human spinal cord after traumatic central nervous system injury. J Neurotrauma 32:1393-1402.

5. Lacroix S, Hamilton LK, Vaugeois A, Beaudoin S, BreaultDugas C, Pineau I, Lévesque SA, Grégoire CA, Fernandes KJ
(2014) Central canal ependymal cells proliferate extensively in response to traumatic spinal cord injury but not demyelinating lesions. PLoS One 9:e85916.

6. Meletis K, Barnabé-Heider F, Carlén M, Evergren E, Tomilin N, Shupliakov O, Frisén J (2008) Spinal cord injury reveals multilineage differentiation of ependymal cells. PLoS Biol 6:e182.

7. Piltti KM, Avakian SN, Funes GM, Hu A, Uchida N, Anderson AJ, Cummings BJ (2015) Transplantation dose alters the dynamics of human neural stem cell engraftment, proliferation and migration after spinal cord injury. Stem Cell Res 15:341353.

8. Busch SA, Hamilton JA, Horn KP, Cuascut FX, Cutrone R, Lehman N, Deans RJ, Ting AE, Mays RW, Silver J (2011) Multipotent adult progenitor cells prevent macrophage-mediated axonal dieback and promote regrowth after spinal cord injury. J Neurosci 31:944-953.

9. Ma Y, Deng M, Liu M (2019) Effect of differently polarized macrophages on proliferation and differentiation of ependymal cells from adult spinal cord. J Neurotrauma 36:23372347.

10. Silva DF, Esteves AR, Oliveira CR, Cardoso SM (2017) Mitochondrial metabolism power SIRT2-dependent deficient traffic causing Alzheimer's-disease related pathology. Mol Neurobiol 54:4021-4040.

11. Esteves AR, Arduíno DM, Silva DF, Viana SD, Pereira FC, Cardoso SM (2018) Mitochondrial metabolism regulates microtubule acetylome and autophagy trough sirtuin-2: impact for Parkinson's disease. Mol Neurobiol 55:1440-1462.

12. Maxwell MM, Tomkinson EM, Nobles J, Wizeman JW, Amore AM, Quinti L, Chopra V, Hersch SM, Kazantsev AG (2011) The Sirtuin 2 microtubule deacetylase is an abundant neuronal protein that accumulates in the aging CNS. Hum Mol Genet 20:3986-3996.

13. Jeong SG, Cho GW (2017) The tubulin deacetylase sirtuin-2 regulates neuronal differentiation through the ERK/CREB signaling pathway. Biochem Biophys Res Commun 482:182187.

14. McCreedy DA, Wilems TS, Xu H, Butts JC, Brown CR, Smith AW, Sakiyama-Elbert SE (2014) Survival, differentiation, and migration of high-purity mouse embryonic stem cell-derived progenitor motor neurons in fibrin scaffolds after sub-acute spinal cord injury. Biomater Sci 2:1672-1682.

15. Wei L, Fraser JL, Lu ZY, Hu X, Yu SP (2012) Transplantation of hypoxia preconditioned bone marrow mesenchymal stem cells enhances angiogenesis and neurogenesis after cerebral 
ischemia in rats. Neurobiol Dis 46:635-645.

16. Khazaei M, Ahuja CS, Fehlings MG (2017) Induced pluripotent stem cells for traumatic spinal cord injury. Front Cell Dev Biol 4:152.

17. Kumamaru H, Saiwai H, Kubota K, Kobayakawa K, Yokota K, Ohkawa Y, Shiba K, Iwamoto Y, Okada S (2013) Therapeutic activities of engrafted neural stem/precursor cells are not dormant in the chronically injured spinal cord. Stem Cells 31:1535-1547.

18. Alfaro-Cervello C, Soriano-Navarro M, Mirzadeh Z, AlvarezBuylla A, Garcia-Verdugo JM (2012) Biciliated ependymal cell proliferation contributes to spinal cord growth. J Comp Neurol 520:3528-3552.

19. Pfenninger CV, Steinhoff C, Hertwig F, Nuber UA (2011) Prospectively isolated CD133/CD24-positive ependymal cells from the adult spinal cord and lateral ventricle wall differ in their long-term in vitro self-renewal and in vivo gene expression. Glia 59:68-81.

20. Moreno-Manzano V, Rodríguez-Jiménez FJ, García-Roselló M, Laínez S, Erceg S, Calvo MT, Ronaghi M, Lloret M, Planells-Cases R, Sánchez-Puelles JM, Stojkovic M (2009) Activated spinal cord ependymal stem cells rescue neurological function. Stem Cells 27:733-743.

21. Ren Y, Ao Y, O'Shea TM, Burda JE, Bernstein AM, Brumm AJ, Muthusamy N, Ghashghaei HT, Carmichael ST, Cheng L, Sofroniew MV (2017) Ependymal cell contribution to scar formation after spinal cord injury is minimal, local and dependent on direct ependymal injury. Sci Rep 7:41122.

22. Spejo AB, Chiarotto GB, Ferreira ADF, Gomes DA, Ferreira RS Jr, Barraviera B, Oliveira ALR (2018) Neuroprotection and immunomodulation following intraspinal axotomy of motoneurons by treatment with adult mesenchymal stem cells. J Neuroinflammation 15:230.

23. Dooley D, Lemmens E, Vangansewinkel T, Le Blon D, Hoornaert C, Ponsaerts P, Hendrix S (2016) Cell-based delivery of interleukin-13 directs alternative activation of macrophages resulting in improved functional outcome after spinal cord injury. Stem Cell Reports 7:1099-1115.

24. Fourcade S, Morató L, Parameswaran J, Ruiz M, Ruiz-Cortés T, Jové M, Naudí A, Martínez-Redondo P, Dierssen M, Ferrer I, Villarroya F, Pamplona R, Vaquero A, Portero-Otín M, Pujol A (2017) Loss of SIRT2 leads to axonal degeneration and locomotor disability associated with redox and energy imbalance. Aging Cell 16:1404-1413.

25. Yang M, Peng Y, Liu W, Zhou M, Meng Q, Yuan C (2019) Sirtuin 2 expression suppresses oxidative stress and senescence of nucleus pulposus cells through inhibition of the p53/p21 pathway. Biochem Biophys Res Commun 513:616-622.

26. Eshun-Wilson L, Zhang R, Portran D, Nachury MV, Toso DB, Löhr T, Vendruscolo M, Bonomi M, Fraser JS, Nogales E (2019) Effects of a-tubulin acetylation on microtubule structure and stability. Proc Natl Acad Sci U S A 116:10366-10371.

27. Bowne-Anderson H, Zanic M, Kauer M, Howard J (2013) Microtubule dynamic instability: a new model with coupled GTP hydrolysis and multistep catastrophe. Bioessays 35:452461.

28. Creppe C, Malinouskaya L, Volvert ML, Gillard M, Close P, Malaise O, Laguesse S, Cornez I, Rahmouni S, Ormenese S, Belachew S, Malgrange B, Chapelle JP, Siebenlist U, Moonen G, Chariot A, Nguyen L (2009) Elongator controls the migration and differentiation of cortical neurons through acetylation of alpha-tubulin. Cell 136:551-564.

29. Szegő ÉM, Gerhardt E, Outeiro TF (2017) Sirtuin 2 enhances dopaminergic differentiation via the AKT/GSK-3 $\beta / \beta$-catenin pathway. Neurobiol Aging 56:7-16.

30. Li XT, Liang Z, Wang TT, Yang JW, Ma W, Deng SK, Wang XB, Dai YF, Guo JH, Li LY (2017) Brain-derived neurotrophic factor promotes growth of neurons and neural stem cells possibly by triggering the phosphoinositide 3-kinase/ AKT/ glycogen synthase kinase-3 $\beta / \beta$-catenin pathway. CNS Neurol Disord Drug Targets 16:828-836.

31. Hachem LD, Mothe AJ, Tator CH (2015) Effect of BDNF and other potential survival factors in models of in vitro oxidative stress on adult spinal cord-derived neural stem/progenitor cells. Biores Open Access 4:146-159.

32. Assaife-Lopes N, Sousa VC, Pereira DB, Ribeiro JA, Sebastião AM (2014) Regulation of TrkB receptor translocation to lipid rafts by adenosine $\mathrm{A}(2 \mathrm{~A})$ receptors and its functional implications for BDNF-induced regulation of synaptic plasticity. Purinergic Signal 10:251-267.

33. Ricci S, Viggiano D, Cimmino I, Perruolo G, Cabaro S, Liotti A, Fiory F, Spinelli R, Di Carlo A, Beguinot F, Formisano P, Oriente F (2018) Prepl deficiency affects olfactory perception and feeding behavior by impairing BDNF-TrkB mediated neurotrophic signaling. Mol Neurobiol 55:6801-6815.

34. Silva-Peña D, Rivera P, Alén F, Vargas A, Rubio L, GarcíaMarchena N, Pavón FJ, Serrano A, Rodríguez de Fonseca F, Suárez J (2019) Oleoylethanolamide modulates BDNF-ERK signaling and neurogenesis in the hippocampi of rats exposed to $\triangle 9$-THC and ethanol binge drinking during adolescence. Front Mol Neurosci 12:96.

35. Liu M, Kay JC, Shen S, Qiao LY (2015) Endogenous BDNF 
augments NMDA receptor phosphorylation in the spinal cord via PLC $\gamma$, PKC, and PI3K/Akt pathways during colitis. J Neuroinflammation 12:151.

36. Zanin JP, Montroull LE, Volosin M, Friedman WJ (2019) The p75 neurotrophin receptor facilitates TrkB signaling and function in rat hippocampal neurons. Front Cell Neurosci 13:485. 